\title{
Physicochemical Characteristics of Waterholes and Soil in a Wildlife Park: A Case Study of Old Oyo National Park, Nigeria
}

\author{
${ }^{1}$ ADETUGA, AT ${ }^{1},{ }^{1}$ OMONONA, AO; ${ }^{2 *}$ JUBRIL, AJ \\ ${ }^{I}$ Department of Wildlife and Ecotourism Management, University of Ibadan, Ibadan. Nigeria \\ ${ }^{2}$ Department of Veterinary Pathology, University of Ibadan, Ibadan. Nigeria \\ ${ }^{*}$ Corresponding Author Email: afusatjagun@yahoo.com
}

\begin{abstract}
Analysing the physicochemical characteristics of water and soil in conservation areas gives information about their quality and the effective management strategies wildlife conservationists could adopt. The study assessed the physicochemical characteristics of selected perennial rivers and soil samples in Old Oyo National Park, Nigeria. A total of 24 composited surface water and 36 topsoil samples were collected using grab and random sampling techniques, respectively for four seasons (two dry and two wet) for two consecutive years (2017 and 2018). The samples were analysed for selected physicochemical characteristics using standard methods. Data collected were subjected to descriptive and inferential (ANOVA) statistics SPSS (version 20.0) at $\alpha_{0.05}$. The result showed that the mean values (in the water samples) of Total Suspended Solids $(673.13 \pm 592.10)$ in dry season 2018, Total Solids $(799.37 \pm 610.17)$ in dry season 2018, and Sulphate (469.34 354.94$)$ in dry season 2017 were above the comparable WHO permissible limit while the mean values (in the soil samples) of total nitrogen (except dry season 2018), exchangeable Magnesium and Potassium (across all the seasons) were above the comparable critical limits. There were significant differences in all the physicochemical characteristics of water sampled [except $\mathrm{pH}(\mathrm{P}=0.12)$, chloride $(\mathrm{P}=0.96)$ and $\mathrm{BOD}(\mathrm{P}=0.86)$ ] while organic carbon $(\mathrm{P}=0.047)$, organic matter $(\mathrm{P}=0.041)$, nitrogen $(\mathrm{P}=0.020)$, calcium $(\mathrm{P}=0.016)$, total exchangeable bases $(\mathrm{P}=0.009)$ and effective cation exchange capacity $(\mathrm{P}=0.033)$ in soils were significantly different across the seasons of sampling. The soil physicochemical parameters above comparable critical limits may have elicited from the impact of anthropogenic activities by the surrounding communities with possible implication on wild animal health in the park.
\end{abstract}

\section{DOI: https://dx.doi.org/10.4314/jasem.v25i6.7}

Copyright: Copyright $\odot 2021$ Adetuga et al. This is an open access article distributed under the Creative Commons Attribution License (CCL), which permits unrestricted use, distribution, and reproduction in any medium, provided the original work is properly cited.

Dates: Received: 20 March 2021; Revised: 27 April 2021; Accepted: 07 May 2021

Keywords: Physicochemical characteristics, water quality, soil, Old Oyo National Park.

Water is one of the most important and essential natural resources that exists on our planet and is essential for survival of both aquatic and terrestrial organisms (Swaleh and Usmani, 2016). The importance of water as a resource is not only tied to its availability and quantity but also to its quality. The quality of water is often generally explained in terms of its physicochemical factors and biological characteristics (Diersing, 2009) and also in terms of its composition rather than its level, volume or flow which are collectively referred to as water quantity (Davies-Colley, 2013). The quality of water within an ecosystem gives salient information about the available resources for supporting life in that ecosystem (Ajibade et al., 2008). Physicochemical parameters have been reported to affect the biotic components of an aquatic environment in various ways (Ayoade et al., 2006). Therefore, the analysis of the physicochemical parameters of water is necessary to understand ecological and environmental pathways of aquatic resources (Patil et al., 2012).

Soil is a complex and dynamic ecosystem whose functionality is related to the links that exist between physical, chemical, and biological properties as well as resident microbial communities (Furtak et al., 2019). It is an important abiotic component of the environment whose availability is crucial to the existence of living organisms. Soils are often considered as one of the most important ecological factors (Gothwal and Gupta, 2019). Its role therefore in determining the optimal productivity of the terrestrial ecosystem cannot be overemphasized (Dar et al., 2018) as it is a vital component as well as medium of unconsolidated nutrients and materials, forming the life layer of plants (Chaudhari, 2013). The quality of soil in a given environment is often defined by its physical, chemical, biochemical as well as microbial characteristics (Furtak and Gajda, 2018). The proper management of soil quality plays an important role in protecting the environment, through preserving biodiversity and good agricultural practices (Lemaire et al., 2014). Even though plant composition may alter the soil physical and chemical properties, which in turn disturbs land efficiency (Singh et al., 2011), their growth depends on the physicochemical properties and organic matter content of the soil to a very large extent (Alsumaiti et al., 2018). 
As far as wildlife management is concerned, the main objective of estimation of water quality criteria is to protect wildlife health in the environment. The interactions of both the physical and chemical characteristics of water and soil play a significant role in composition, abundance, movement and diversity of aquatic species and soil organisms (Deepak and Singh, 2014; Edori and Iyama, 2017). This study therefore aimed at assessing the physicochemical characteristics of selected waterholes and soil samples in Old Oyo National Park and also checked if seasonal variation had influential impacts on the parameters assessed.

\section{MATERIALS AND METHODS}

Study Area: The Old Oyo National Park (OONP) previously occurred as two contiguous forest reserves; Upper Ogun and Oyo-Ile which were gazetted in 1936 and 1941, respectively (Oyeleke et al., 2015). The park has a total land mass area of $2,512 \mathrm{~km}^{2}$ making it the fourth largest national park in Nigeria (Oladeji et al., 2012). It is located between latitudes $8^{0} 15^{\circ}$ and $9^{0}$ $05^{`} \mathrm{~N}$ and longitudes $3^{\circ} 35^{`}$ and $4^{\circ} 42^{`} \mathrm{E}$, and centered on North latitude $8^{\circ} 36^{\prime} 00^{\prime \prime}$ and East longitude $3^{\circ} 57^{\prime}$ 05" (Akinyemi and Kayode, 2010). The park is situated in a transition vegetation zones between mixed deciduous rainforest ecosystem and open savannah woodlands in the north. The annual rainfall varies between $1110 \mathrm{~mm}$ and $1250 \mathrm{~mm}$ while temperature ranges between $20^{\circ} \mathrm{C}$ and $33.6^{\circ} \mathrm{C}$ (Adetoro et al., 2011). The topography of most part of the park is plain land ranging from $305 \mathrm{~m}-380 \mathrm{~m}$ above sea level. The park is situated in a transition vegetation zones between mixed deciduous rainforest ecosystem and open savannah woodlands in the north. The park lies on crystalline acid rocks with predominantly sandy soils that are derived from basement complex materials (Alarape, 2002). The park is also abundantly rich in flora and fauna species.

Sample Site Selection: The study was carried out within three (3) out of the five ranges of Old Oyo National Park. The ranges include Oyo-Ile, Tede, and Marguba. These ranges were purposively selected based on the presence of perennial waterholes, representativeness of the park and dominant anthropogenic activities by the surrounding local communities such as agriculture, charcoal production, illegal mining and grazing sequel to a thorough reconnaissance survey of the park.

Sample Collection Technique: Water samples were collected from River Owu (in Tede range), Rivers Ogun, Oopo, Ayinta (in Marguba range), Rivers Tessi and Sooro (in Oyo-Ile range) using grab sampling technique. The water samples were collected into sample bottles from different sampling points (upper, middle and lower courses) along the rivers (and composited) and were subsequently analyzed while the means of the replicates were appropriately recorded. A total of twenty-four (24) composited water samples were collected throughout the period of sampling. Also, representative surface soil samples (0 $-15 \mathrm{~cm}$ depth) were randomly collected at three points (Upper slope, Middle slope and Lower slope) along chosen topographical catena (about $1.2 \mathrm{~km}$ long) using a soil auger into well labeled polyethene bags prior to routine soil analysis. Three topographical catenae spaced at $2-5 \mathrm{~km}$ were selected in each selected range. Thus, a total of thirty-six (36) soil samples were collected from the selected ranges throughout the period of sampling. Samples were collected within four seasons for two consecutive years between January 2017 and June, 2018. The water samples were subsequently transported with the aid of an ice-chest box to the Geo-Environmental Research Centre Laboratory, University of Ibadan, Ibadan, Nigeria for water analysis while the soil samples were taken to the Soil Chemistry and Physics Laboratories of the University of Ibadan, Ibadan, Nigeria for soil analysis.

Parameters Evaluated: Water physicochemical characteristics of the samples such as Temperature, $\mathrm{pH}$, Electrical Conductivity (EC), Total Dissolved Solid (TDS), Total Suspended Solids (TSS), Total Solid (TS), Dissolved Oxygen (DO), Chemical Oxygen Demand (COD), Biological Oxygen Demand (BOD), Alkali, Nitrate $\left(\mathrm{NO}_{3}{ }^{-}\right)$, Chloride $\left(\mathrm{Cl}^{-}\right)$, Phosphate $\left(\mathrm{PO}_{4}{ }^{3-}\right)$ and Sulphate $\left(\mathrm{SO}_{4}{ }^{2-}\right)$ and soil physicochemical parameters such as particle size, $\mathrm{pH}$ (water), electrical conductivity (EC), soil organic carbon (SOC), soil organic matter (SOM), soil nitrogen, available phosphorus exchangeable bases ( $\mathrm{Ca}, \mathrm{Mg}, \mathrm{K}, \mathrm{Na}$ ), total exchangeable bases (TEB), exchangeable acidity (EA), effective cation exchange capacity (ECEC), basal saturation (BS) and textural class were evaluated.

Laboratory Analysis of Water and Soil Samples: The $\mathrm{pH}, \mathrm{EC}$, temperature and TDS of all the water samples were determined in-situ (on-site). The $\mathrm{pH}$ was determined using a $\mathrm{pH}$ meter (HI98128 pHep®5 Model) while the EC was measured with conductivity meter (HI9831DiST®5 Model) after calibration at $25^{\circ} \mathrm{C}$. The temperature (sample and ambient) were measured using thermometer (COM-100 Model) while the TDS was determined using a TDS meter (HI9831DiST®5 Model) after calibration at $25^{\circ} \mathrm{C}$. The total suspended solid (TSS) was determined by photometric method using a spectrophotometer at a wavelength of $810 \mathrm{~nm}$ while the total solid (TS) was estimated as the sum total of the suspended solid 
particles (TSS) and dissolved materials (TDS). The alkalinity and chloride levels were determined by titrimetry while phosphate, sulphate and nitrate were determined by colorimetric method, turbidimetry and phenoldisulphonic acid methods, respectively.

\begin{tabular}{|c|c|c|c|}
\hline $\begin{array}{l}\text { Sampling } \\
\text { Ranges }\end{array}$ & $\begin{array}{l}\text { Sample } \\
\text { Codes }\end{array}$ & Sample Code Name & $\begin{array}{l}\text { Coordinates } \\
\text { (latitude/longitude) }\end{array}$ \\
\hline \multirow[t]{3}{*}{ Marguba } & MS1 & $\begin{array}{l}\text { Composited Soil Sample } \\
\text { l in Marzuba Range }\end{array}$ & $\begin{array}{l}\mathrm{N} 08^{\circ} 30^{\circ} 04.6 \\
\mathrm{E} 003^{\circ} 44^{\prime} 34.2\end{array}$ \\
\hline & MS2 & $\begin{array}{l}\text { Composited Soil Sample } \\
2 \text { in Marguba Range }\end{array}$ & $\begin{array}{l}\text { N } 08^{\circ} 29^{\circ} 49.2^{\prime \prime} \\
\text { E } 003^{\circ} 54^{1} 18^{\prime \prime}\end{array}$ \\
\hline & MS3 & $\begin{array}{l}\text { Composited Soil Sample } \\
3 \text { in Marguba Range }\end{array}$ & $\begin{array}{l}\text { N } 08^{\circ} 26^{\prime} 52.3^{\prime \prime} \\
\text { E } 003^{\circ} 59^{\prime} 13.1^{\prime \prime}\end{array}$ \\
\hline \multirow[t]{3}{*}{ Oyo-Ile } & OS1 & $\begin{array}{l}\text { Composited Soil Sample } \\
1 \text { in Oyo-Ile Range }\end{array}$ & $\begin{array}{l}\text { N } 08^{0} 58.296^{\prime} \\
\text { E } 003^{\circ} 57.543\end{array}$ \\
\hline & OS2 & $\begin{array}{l}\text { Composited Soil Sample } \\
2 \text { in Oyo-Ile Range }\end{array}$ & $\begin{array}{l}\text { N } 08^{0} 54.827^{\prime} \\
\text { E } 004^{\circ} 00.736^{\prime}\end{array}$ \\
\hline & OS 3 & $\begin{array}{l}\text { Composited Soil Sample } \\
3 \text { in Oyo-Ile Range }\end{array}$ & $\begin{array}{l}\text { N } 08^{0} 57.589^{\prime} \\
\text { E } 003^{\circ} 58.280^{\prime}\end{array}$ \\
\hline \multirow[t]{3}{*}{ Tede } & TS1 & $\begin{array}{l}\text { Composited Soil Sample } \\
1 \text { in Tede Range }\end{array}$ & $\begin{array}{l}\text { N } 08^{\circ} 27^{\prime} 39.6^{\prime \prime} \\
\text { E } 003^{\circ} 36^{\prime} 46.2^{\prime \prime}\end{array}$ \\
\hline & TS2 & $\begin{array}{c}\text { Composited Soil Sample } \\
2 \text { in Tede Range }\end{array}$ & $\begin{array}{l}\text { N } 08^{\circ} 29^{\prime} 00.1^{\prime \prime} \\
\text { E } 003^{\circ} 33^{\prime} 56.4^{\prime \prime}\end{array}$ \\
\hline & TS3 & $\begin{array}{c}\text { Composited Soil Sample } \\
3 \text { in Tede Range }\end{array}$ & $\begin{array}{l}\text { N } 08^{\circ} 16^{\prime} 22.8^{\circ} \\
\text { E } 003^{\circ} 44^{\prime} 56.4^{\circ}\end{array}$ \\
\hline
\end{tabular}

The DO was determined using the azide modification of the Winkler titration method while the BOD as well as COD were also determined using the titration method. Soil particle size distribution was determined using the Bouyoucos hydrometer method (Gee and Or, 2002). The soil $\mathrm{pH}$ was determined with the $\mathrm{pH}$ meter using glass electrode in a 1:1 soil to water ratio (Udo et al., 2009). The electrical conductivity of the soil samples was determined in the filtrate of the water extracts by making use of a conductivity meter. Total nitrogen was determined by Kjehdahl digestion method (Bremmer, 1996). Soil organic carbon was determined using the Walkey Black wet oxidation method (Nelsen and Sommers, 1982). Organic matter of sampled soils was obtained by multiplying \% Organic carbon with conventional 'van Bemmelar factor of 1.724. Available phosphorus was determined with spectrophotometer using Mehlich III as extractant (Jackson, 1958). Exchangeable bases were determined using neutral NH OAC 4 leachate. Exchangeable $\mathrm{Ca}$ and $\mathrm{Mg}$ were determined by EDTA versanate titration method (McLean, 1982). Exchangeable $\mathrm{Na}$ and $\mathrm{K}$ were determined by the flame photometer method while exchangeable acidity was determined by leaching the soil with $1 \mathrm{~N} \mathrm{KCl}$ and titrating with $0.05 \mathrm{~N} \mathrm{NaOH}$ (McLean, 1982).

Statistical Analysis: Data collected were subjected to descriptive (mean, standard deviation) and inferential (ANOVA) statistics while post-hoc test (LSD) was used to determine significant differences across the seasons of sampling with statistical significance set at $\alpha_{0.05}$. All the statistical analyses were performed with SPSS software (version 20.0).

\section{RESULTS AND DISCUSSION}

Physicochemical Characteristics of water Samples: The result of dry season, 2017 showed that the sampled rivers' temperatures (except River Ogun), sulphate levels in all the rivers (except Rivers Oopo and Owu) and the electrical conductivity (River Ogun) were above the WHO permissible limit as shown in Table 2 while in the wet season of 2017 , only the $\mathrm{pH}$ of River Sooro was above the WHO permissible limit (Table 3). The result of dry season, 2018 showed that the sampled rivers' temperatures (except River Ogun), sulphate levels in all the rivers (except Rivers Ayinta and Owu) and the total solid (except Rivers Ayinta and Owu) were above the WHO permissible limit as shown in Table 4 while during the wet season of 2018 , all the physicochemical parameters analysed in all the sampled rivers were below the WHO permissible limit as shown in Table 5. Of noteworthy is the fact that the mean levels of physicochemical parameters ( $\mathrm{pH}, \mathrm{EC}$, TDS, TSS, TS, sulphate and chloride) during the dry seasons were higher than those of the wet seasons while statistically, there were significant differences in all the physicochemical parameters of water sampled except $\mathrm{pH}$, chloride and BOD that had no significant differences (Table 6). Figure 1 shows the mean plot of water physicochemical parameters that were above the permissible limits. The result shows that the Total Suspended Solid (TSS), Total Solids (TS) and sulphate levels were highest in Tede range, followed by Marguba range and least in Oyo-Ile range.

Physicochemical Characteristics of Sampled Soils: The result showed that during the dry season of 2017, the \% N (in MS1, OS3, TS1 and TS3), Mg (in all the samples) and $\mathrm{K}$ (in all the samples except OS3) were above the comparable critical limit as shown in Table 7. During the wet season of 2017, in all the soil samples, the \% $\mathrm{N}$ (except OS3), Mg (in all the samples) as well as K (in all the samples except OS1, OS2 and OS3) were above the critical limit as shown in Table 8 . In the dry season of 2018, in all the soil samples, \% N (except in MS2, OS1, OS2, OS3) and exchangeable bases [Mg, $\mathrm{K}$ (except in OS3) were above the critical limit as shown in Table 9. During the wet season of 2018, in all the soil samples, the \% $\mathrm{N}$ (except OS1, OS2, OS3) and exchangeable bases (Mg, $\mathrm{K}$ [except OS3]) as shown in Table 10 were above the critical limit. The mean values of most of the physicochemical parameters of soil samples from Old Oyo National Park across the four seasons of sampling are shown in Table 11 with \% N (except dry season 2018), Mg (in all the seasons) and $\mathrm{K}$ (in all the seasons) were above the comparable critical limits.. 


\begin{tabular}{|c|c|c|c|c|c|c|c|c|c|c|c|c|c|c|c|}
\hline $\begin{array}{l}\text { Water } \\
\text { Holes }\end{array}$ & $\begin{array}{l}\text { Temp } \\
\left({ }^{\circ} \mathrm{C}\right)\end{array}$ & $\begin{array}{l}\text { Temp } \\
\left({ }^{\circ} \mathrm{C}\right)\end{array}$ & pH & $\begin{array}{l}\text { EC } \\
(\mu \mathrm{S} / \mathrm{cm})\end{array}$ & $\begin{array}{l}\text { Alkal. } \\
\text { (mg/l) }\end{array}$ & $\begin{array}{l}\text { TDS } \\
(\mathbf{m g} / \mathbf{l})\end{array}$ & $\begin{array}{l}\text { TSS } \\
(\mathbf{m g} / \mathbf{l})\end{array}$ & $\begin{array}{l}\text { TS } \\
(\mathbf{m g} / \mathbf{l})\end{array}$ & $\begin{array}{l}\mathrm{NO}_{3} \\
(\mathrm{mg} / \mathrm{l})\end{array}$ & $\begin{array}{l}\mathrm{PO}_{4}{ }^{3-} \\
(\mathrm{mg} / \mathrm{l})\end{array}$ & $\begin{array}{l}\mathrm{SO}_{4}{ }^{2-} \\
(\mathrm{mg} / \mathrm{l})\end{array}$ & $\begin{array}{l}\mathrm{Cl}^{-} \\
(\mathbf{m g} / \mathbf{l})\end{array}$ & $\begin{array}{l}\text { DO } \\
(\mathrm{mg} /)\end{array}$ & $\begin{array}{l}\text { BOD } \\
(\mathrm{mg} / \mathrm{l})\end{array}$ & $\begin{array}{l}\text { COD } \\
\text { (mg/l) }\end{array}$ \\
\hline River Ogun & 28.22 & 25.32 & 7.10 & 270.00 & 71.20 & 173.0 & 112.0 & 285.0 & 0.19 & 0.005 & 789.12 & 16.34 & 7.44 & 5.43 & 36.14 \\
\hline River Oopo & 23.81 & 20.46 & 6.51 & 150.00 & 54.14 & 147.4 & 158.0 & 305.4 & 0.07 & 0.004 & 102.11 & 26.08 & 7.22 & 23.76 & 48.26 \\
\hline River Ayinta & 22.62 & 18.20 & 6.67 & 203.00 & 48.16 & 147.0 & 193.0 & 340.0 & 0.37 & 0.026 & 408.08 & 14.11 & 4.06 & 15.52 & 29.48 \\
\hline River Tessi & 24.32 & 19.22 & 6.84 & 220.00 & 33.54 & 141.0 & 344.6 & 485.6 & 0.79 & 0.039 & 987.47 & 9.16 & 2.21 & 8.96 & 32.51 \\
\hline River Sooro & 15.68 & 18.00 & 6.56 & 203.00 & 68.20 & 147.2 & 137.8 & 285.0 & 0.55 & 0.007 & 400.22 & 15.92 & 2.74 & 5.98 & 14.64 \\
\hline River Owu & 27.30 & 24.10 & 6.87 & 80.10 & 52.04 & 51.2 & 33.2 & 84.4 & 0.05 & 0.003 & 129.04 & 2.64 & 4.12 & 24.67 & 48.11 \\
\hline
\end{tabular}

Table 3: Physicochemical Parameters of selected waterholes in Old Oyo National Park [Wet Season, 2017]

\begin{tabular}{|c|c|c|c|c|c|c|c|c|c|c|c|c|c|c|c|}
\hline $\begin{array}{l}\text { Water } \\
\text { Holes }\end{array}$ & $\begin{array}{l}\text { A Temp } \\
\left({ }^{\circ} \mathrm{C}\right)\end{array}$ & $\begin{array}{l}\text { S. Temp } \\
\left({ }^{\circ} \mathrm{C}\right)\end{array}$ & pH & $\begin{array}{l}\mathrm{EC} \\
(\mu \mathrm{S} / \mathrm{cm})\end{array}$ & $\begin{array}{l}\text { Alkal. } \\
\text { (mg/l) }\end{array}$ & $\begin{array}{l}\text { TDS } \\
\text { (mg/l) }\end{array}$ & $\begin{array}{l}\text { TSS } \\
\text { (mg/l) }\end{array}$ & $\begin{array}{l}\text { TS } \\
(\mathrm{mg} / \mathrm{l})\end{array}$ & $\begin{array}{l}\mathrm{NO}_{3} \\
(\mathrm{mg} /)\end{array}$ & $\begin{array}{l}\mathrm{PO}_{4}{ }^{3-} \\
(\mathrm{mg} / \mathrm{l})\end{array}$ & $\begin{array}{l}\mathrm{SO}_{4}{ }^{2-} \\
(\mathrm{mg} / \mathrm{l})\end{array}$ & $\begin{array}{l}\mathrm{Cl}^{-} \\
(\mathrm{mg} / \mathrm{l})\end{array}$ & $\begin{array}{l}\text { DO } \\
(\mathrm{mg} /)\end{array}$ & $\begin{array}{l}\text { BOD } \\
(\mathrm{mg} / \mathrm{l})\end{array}$ & $\begin{array}{l}\text { COD } \\
(\mathrm{mg} / \mathrm{l})\end{array}$ \\
\hline River Ogun & 23.60 & 25.10 & 6.93 & 72.50 & 80.00 & 36.70 & 224.30 & 261.00 & 0.50 & 0.24 & 64.70 & 9.93 & 5.50 & 8.00 & 57.90 \\
\hline River Oopo & 25.10 & 25.40 & 7.01 & 77.80 & 60.00 & 39.20 & 163.80 & 203.00 & 0.56 & 0.26 & 60.60 & 17.90 & 6.70 & 9.00 & 50.80 \\
\hline River Ayinta & 27.50 & 26.20 & 6.47 & 82.40 & 70.00 & 46.00 & 218.00 & 264.00 & 0.43 & 0.27 & 82.40 & 16.20 & 6.54 & 8.00 & 56.20 \\
\hline River Tessi & 28.10 & 28.10 & 6.53 & 48.80 & 50.00 & 24.90 & 103.10 & 128.00 & 0.31 & 0.28 & 54.10 & 15.90 & 6.90 & 16.00 & 43.80 \\
\hline River Sooro & 28.10 & 27.05 & 6.43 & 75.00 & 80.00 & 38.10 & 130.90 & 169.00 & 0.50 & 0.29 & 28.40 & 21.80 & 6.40 & 24.00 & 87.70 \\
\hline River Owu & 26.50 & 25.83 & 6.72 & 80.00 & 70.00 & 40.40 & 94.60 & 135.00 & 0.17 & 0.230 & 57.80 & 5.96 & 6.90 & 28.00 & 53.80 \\
\hline
\end{tabular}

Table 4: Physicochemical Parameters of selected waterholes in Old Oyo National Park [Dry Season, 2018]

\begin{tabular}{|c|c|c|c|c|c|c|c|c|c|c|c|c|c|c|c|}
\hline $\begin{array}{l}\text { Water } \\
\text { Holes }\end{array}$ & $\begin{array}{l}\text { A Temp } \\
\left({ }^{\circ} \mathrm{C}\right)\end{array}$ & $\begin{array}{l}\text { S. Temp } \\
\left({ }^{\circ} \mathrm{C}\right)\end{array}$ & pH & $\begin{array}{l}\text { EC } \\
(\mu \mathrm{S} / \mathrm{cm})\end{array}$ & $\begin{array}{l}\text { Alkal. } \\
\text { (mg/l) }\end{array}$ & $\begin{array}{l}\text { TDS } \\
(\mathrm{mg} / \mathrm{l})\end{array}$ & $\begin{array}{l}\text { TSS } \\
(\mathrm{mg} / \mathrm{l})\end{array}$ & $\begin{array}{l}\text { TS } \\
(\mathrm{mg} / \mathrm{l})\end{array}$ & $\begin{array}{l}\mathrm{NO}_{3} \\
(\mathbf{m g} /)\end{array}$ & $\begin{array}{l}\mathrm{PO}_{4}{ }^{3-} \\
(\mathrm{mg} / \mathrm{l})\end{array}$ & $\begin{array}{l}\mathrm{SO}_{4}{ }^{2-} \\
(\mathrm{mg} / \mathrm{l})\end{array}$ & $\begin{array}{l}\mathrm{Cl}^{-} \\
(\mathrm{mg} / \mathrm{l})\end{array}$ & $\begin{array}{l}\text { DO } \\
(\mathbf{m g} /)\end{array}$ & $\begin{array}{l}\text { BOD } \\
(\mathrm{mg} / \mathrm{l})\end{array}$ & $\begin{array}{l}\text { COD } \\
(\mathrm{mg} / \mathrm{l})\end{array}$ \\
\hline River Ogun & 25.50 & 26.12 & 6.82 & 210.26 & 86.56 & 163.00 & 340.34 & 503.34 & 0.23 & 0.063 & 14.33 & 10.90 & 5.90 & 8.05 & 28.20 \\
\hline River Oopo & 24.44 & 22.20 & 6.74 & 174.15 & 43.38 & 152.23 & 1675.0 & 1827.0 & 0.01 & 0.217 & 85.72 & 37.71 & 5.30 & 30.20 & 120.14 \\
\hline River Ayinta & 24.20 & 23.28 & 6.71 & 196.10 & 57.23 & 126.50 & 206.00 & 332.50 & 0.26 & 0.142 & 54.32 & 11.52 & 4.22 & 11.40 & 46.65 \\
\hline River Tessi & 23.94 & 23.10 & 6.72 & 214.12 & 28.82 & 118.40 & 680.05 & 798.45 & 0.20 & 0.198 & 28.64 & 11.92 & 4.50 & 10.18 & 36.35 \\
\hline River Sooro & 20.53 & 19.24 & 6.52 & 218.01 & 56.54 & 134.64 & 1015.0 & 1149.7 & 0.28 & 0.435 & 8.57 & 17.93 & 6.40 & 5.00 & 20.50 \\
\hline River Owu & 25.10 & 23.14 & 6.91 & 88.42 & 64.32 & 63.10 & 122.42 & 185.52 & 0.11 & 0.160 & 37.24 & 3.22 & 5.27 & 19.31 & 43.26 \\
\hline
\end{tabular}

Table 5: Physicochemical Parameters of selected waterholes in Old Oyo National Park [Wet Season, 2018]

\begin{tabular}{|c|c|c|c|c|c|c|c|c|c|c|c|c|c|c|c|}
\hline $\begin{array}{l}\text { Water } \\
\text { Holes }\end{array}$ & $\begin{array}{l}\text { A Temp } \\
\left({ }^{\circ} \mathrm{C}\right)\end{array}$ & $\begin{array}{l}\text { S. Temp } \\
\left({ }^{\circ} \mathrm{C}\right)\end{array}$ & $\mathbf{p H}$ & $\begin{array}{l}\text { EC } \\
(\mu \mathrm{S} / \mathrm{cm})\end{array}$ & $\begin{array}{l}\text { Alkal. } \\
(\mathrm{mg} / \mathrm{l})\end{array}$ & $\begin{array}{l}\text { TDS } \\
(\mathrm{mg} / \mathrm{l})\end{array}$ & $\begin{array}{l}\text { TSS } \\
(\mathrm{mg} / \mathrm{l})\end{array}$ & $\begin{array}{l}\text { TS } \\
(\mathrm{mg} / \mathrm{l})\end{array}$ & $\begin{array}{l}\mathrm{NO}_{3} \\
(\mathrm{mg} / \mathrm{l})\end{array}$ & $\begin{array}{l}\mathrm{PO}_{4}{ }^{3-} \\
(\mathrm{mg} / \mathrm{l})\end{array}$ & $\begin{array}{l}\mathrm{SO}_{4}{ }^{2-} \\
(\mathrm{mg} / \mathrm{l})\end{array}$ & $\begin{array}{l}\mathrm{Cl}^{-} \\
(\mathrm{mg} / \mathrm{l})\end{array}$ & $\begin{array}{l}\text { DO } \\
(\mathrm{mg} /)\end{array}$ & $\begin{array}{l}\text { BOD } \\
(\mathrm{mg} / \mathrm{l})\end{array}$ & $\begin{array}{l}\text { COD } \\
(\mathrm{mg} / \mathrm{l})\end{array}$ \\
\hline River Ogun & 24.20 & 25.18 & 6.68 & 178.10 & 92.50 & 154.1 & 362.4 & 516.50 & 0.34 & 0.074 & 32.14 & 14.31 & 4.75 & 6.45 & 24.53 \\
\hline River Oopo & 23.70 & 22.6 & 6.52 & 105.20 & 37.20 & 158.7 & 293.1 & 451.8 & 0.04 & 0.208 & 88.05 & 24.2 & 6.10 & 28.72 & 68.35 \\
\hline River Ayinta & 25.40 & 25.05 & 6.54 & 98.72 & 62.30 & 149.4 & 304.5 & 453.95 & 0.32 & 0.161 & 62.50 & 9.68 & 3.92 & 7.54 & 38.12 \\
\hline River Tessi & 21.50 & 23.25 & 6.70 & 128.30 & 48.40 & 174.2 & 282.0 & 456.20 & 0.26 & 0.196 & 36.80 & 17.22 & 4.52 & 12.44 & 25.62 \\
\hline River Sooro & 22.10 & 23.07 & 6.48 & 96.50 & 68.30 & 152.8 & 279.2 & 432.0 & 0.26 & 0.473 & 12.42 & 19.1 & 5.53 & 9.41 & 24.74 \\
\hline River Owu & 22.45 & 21.30 & 6.83 & 93.50 & 67.50 & 72.60 & 149.2 & 221.8 & 0.23 & 0.21 & 44.32 & 4.16 & 4.28 & 14.30 & 32.41 \\
\hline
\end{tabular}

The physicochemical characteristics of water samples obtained from this study are comparable with those of typical tropical rivers. The lower values recorded in the dry season could be due to the period / time of sampling which was early in the morning. Seasonal variations in water temperature depend on where they are located (Perlman, 2013) though the increase in water temperature is directly related to total dissolved or suspended solids (Martinez et al., 2011). The temperature of the water samples observed in this study fell within the temperature range recommended for aquatic life in the tropical environment (Olukunle, 2000). Higher values of pH were observed in the dry seasons and this is contrary to the findings of Ajibade et al. (2008) and Omonona et al. (2018). Any change in $\mathrm{pH}$ in water outside the permissible limits may hold dire consequences for the health of aquatic organisms since most of their metabolic activities are $\mathrm{pH}$ dependent (Chen and Lin, 1995). The mean $\mathrm{pH}$ values across the four seasons imply that the water samples (rivers) are acidic and may therefore not be potable or safe for drinking. 
Table 6: Mean values of physicochemical parameters of the selected waterholes of Old Oyo National Park

\begin{tabular}{|c|c|c|c|c|c|c|}
\hline \multirow[b]{2}{*}{ Parameters } & \multicolumn{4}{|c|}{ Mean Values \pm Standard Deviation } & \multirow{2}{*}{$\begin{array}{l}\text { WHO (2011) } \\
\text { Guideline for } \\
\text { Drinking water }\end{array}$} & \multirow{2}{*}{$\begin{array}{l}\text { NSDWQ (2007) } \\
\text { Guideline for } \\
\text { Drinking water }\end{array}$} \\
\hline & $\begin{array}{l}\text { Dry Season } \\
(\text { Jan. 2017) }\end{array}$ & $\begin{array}{l}\text { Wet Season } \\
\text { (June, 2017) } \\
\end{array}$ & $\begin{array}{l}\text { Dry Season } \\
\text { (Jan. 2018) }\end{array}$ & $\begin{array}{l}\text { Wet Season } \\
\text { (May, 2018) }\end{array}$ & & \\
\hline Ambient Temp $\left({ }^{\circ} \mathrm{C}\right)$ & $23.67 \pm 4.46^{\mathrm{ab}}$ & $26.48 \pm 1.82^{\mathrm{abcd}}$ & $23.95 \pm 1.77^{\mathrm{bc}}$ & $23.23 \pm 1.47^{\mathrm{bd}}$ & Ambient & Ambient \\
\hline Sample Temp $\left({ }^{\circ} \mathrm{C}\right)$ & $20.88 \pm 3.11^{\mathrm{abc}}$ & $26.28 \pm 1.12^{\mathrm{abc}}$ & $22.85 \pm 2.21^{\mathrm{ab}}$ & $23.41 \pm 1.49^{\mathrm{ac}}$ & $25-30$ & Ambient \\
\hline $\mathrm{pH}$ & $6.76 \pm 0.22^{\mathrm{a}}$ & $6.68 \pm 0.25$ & $6.74 \pm 0.13$ & $6.63 \pm 0.13^{\mathrm{a}}$ & $6.5-8.5$ & $6.5-8.5$ \\
\hline $\mathrm{EC}(\mu \mathrm{S} / \mathrm{cm})$ & $187.68 \pm 65.24^{\mathrm{ab}}$ & $72.75 \pm 12.25^{\mathrm{abc}}$ & $183.51 \pm 49.26^{\mathrm{bc}}$ & $116.72 \pm 32.58^{\mathrm{abc}}$ & 250 & 1000 \\
\hline Alkalinity (mg/l) & $54.55 \pm 13.80^{\mathrm{ab}}$ & $68.33 \pm 11.69^{\mathrm{abc}}$ & $56.14 \pm 19.51^{\mathrm{bc}}$ & $62.70 \pm 18.96$ & 100 & 100 \\
\hline TDS $(\mathrm{mg} / \mathrm{l})$ & $134.47 \pm 42.30^{\mathrm{ab}}$ & $37.55 \pm 6.98^{\text {abcd }}$ & $126.31 \pm 35.06^{\mathrm{bc}}$ & $143.64 \pm 35.87^{\mathrm{bd}}$ & 500 & 500 \\
\hline $\mathrm{TSS}(\mathrm{mg} / \mathrm{l})$ & $163.10 \pm 103.91^{\mathrm{ac}}$ & $155.78 \pm 56.16^{\mathrm{bc}}$ & $673.13 \pm 592.10^{\text {abcd }}$ & $278.40 \pm 70.23^{\text {cd }}$ & 500 & - \\
\hline $\mathrm{TS}(\mathrm{mg} / \mathrm{l})$ & $297.57 \pm 128.82^{\mathrm{ac}}$ & $193.33 \pm 59.88^{\mathrm{bcd}}$ & $799.37 \pm 610.17^{\text {abcd }}$ & $422.04 \pm 102.16^{\mathrm{bcd}}$ & 500 & 1500 \\
\hline Nitrate $(\mathrm{mg} / \mathrm{l})$ & $0.34 \pm 0.29^{\mathrm{ac}}$ & $0.41 \pm 0.15^{\mathrm{bcd}}$ & $0.18 \pm 0.10^{\mathrm{abc}}$ & $0.24 \pm 0.11^{\mathrm{bd}}$ & 10 & 50 \\
\hline $\mathrm{PO}_{4}^{3-}(\mathrm{mg} / \mathrm{l})$ & $0.01 \pm 0.01^{\mathrm{abcd}}$ & $0.26 \pm 0.02^{\mathrm{abc}}$ & $0.20 \pm 0.13^{\mathrm{abc}}$ & $0.22 \pm 0.14^{\text {ad }}$ & 5.0 & - \\
\hline $\mathrm{SO}_{4}^{2-}(\mathrm{mg} / \mathrm{l})$ & $469.34 \pm 354.94^{\mathrm{abcd}}$ & $58.00 \pm 17.54^{\mathrm{ab}}$ & $38.14 \pm 28.49^{a c}$ & $46.04 \pm 26.26^{\text {ad }}$ & 400 & 100 \\
\hline $\mathrm{Cl}^{-}(\mathrm{mg} / \mathrm{l})$ & $14.04 \pm 7.84$ & $14.62 \pm 5.72$ & $15.53 \pm 11.83$ & $14.78 \pm 7.10$ & 200 & 250 \\
\hline $\mathrm{DO}(\mathrm{mg} / \mathrm{l})$ & $4.63 \pm 2.22^{\mathrm{ab}}$ & $6.49 \pm 0.52^{\text {abcd }}$ & $5.27 \pm 0.82^{\mathrm{bc}}$ & $4.85 \pm 0.82^{\mathrm{bd}}$ & 7.5 & - \\
\hline BOD $(\mathrm{mg} / \mathrm{l})$ & $14.05 \pm 8.66$ & $15.50 \pm 8.76$ & $14.02 \pm 9.26$ & $13.14 \pm 8.18$ & $2.0-6.0$ & - \\
\hline $\mathrm{COD}(\mathrm{mg} / \mathrm{l})$ & $34.86 \pm 12.65^{\mathrm{abc}}$ & $58.37 \pm 15.20^{\mathrm{abd}}$ & $49.18 \pm 36.07^{\mathrm{ac}}$ & $35.63 \pm 16.91^{\mathrm{bd}}$ & 7.5 & - \\
\hline
\end{tabular}

Table 7: Physicochemical Properties of Soil Samples in Old Oyo National Park [Dry Season, 2017]

\begin{tabular}{|c|c|c|c|c|c|c|c|c|c|c|c|c|c|c|c|c|}
\hline \multirow[t]{2}{*}{$\begin{array}{l}\text { Soil } \\
\text { Sample }\end{array}$} & \multirow{2}{*}{$\begin{array}{l}\text { pH } \\
\left(\mathbf{H}_{2} \mathrm{O}\right. \\
\mathbf{1 : 1 )}\end{array}$} & \multirow[t]{2}{*}{$\begin{array}{l}\text { Soil EC } \\
\mu \mathrm{S} / \mathrm{cm})\end{array}$} & \multirow[t]{2}{*}{$\begin{array}{l}\text { SOC } \\
(\%)\end{array}$} & \multirow[t]{2}{*}{$\% \mathrm{~N}$} & \multirow[t]{2}{*}{$\begin{array}{l}\text { A. P } \\
(\mathbf{m g} / \mathbf{k g})\end{array}$} & \multirow[t]{2}{*}{$\begin{array}{l}\text { E.A } \\
\text { (cmol/kg) }\end{array}$} & \multicolumn{4}{|c|}{ Exchangeable bases (cmol/kg) } & \multirow[t]{2}{*}{$\begin{array}{l}\text { TEB } \\
(\mathrm{cmol} / \mathrm{kg})\end{array}$} & \multirow[t]{2}{*}{$\begin{array}{l}\text { ECEC } \\
(\mathrm{cmol} / \mathrm{kg})\end{array}$} & \multirow[t]{2}{*}{ BS (\%) } & \multicolumn{3}{|c|}{$\begin{array}{l}\text { Particle Size } \\
(\mathbf{g} / \mathbf{k g})\end{array}$} \\
\hline & & & & & & & Ca & Mg & $\mathbf{K}$ & $\mathrm{Na}$ & & & & Sand & Silt & Clay \\
\hline MS1 & 6.6 & 108 & 2.54 & 0.26 & 12.90 & 1.60 & 3.70 & 0.95 & 0.68 & 0.36 & 5.69 & 7.29 & 78.05 & 762 & 156 & 82 \\
\hline MS2 & 6.1 & 112 & 0.82 & 0.14 & 13.95 & 1.90 & 3.73 & 0.98 & 0.52 & 0.34 & 5.57 & 7.47 & 74.56 & 824 & 110 & 66 \\
\hline MS3 & 6.0 & 190 & 2.12 & 0.12 & 14.00 & 0.14 & 4.45 & 0.96 & 0.74 & 0.36 & 6.51 & 6.65 & 97.89 & 846 & 56 & 98 \\
\hline OS1 & 6.0 & 118 & 0.59 & 0.08 & 12.40 & 1.75 & 2.68 & 0.72 & 0.82 & 0.52 & 4.74 & 6.49 & 73.04 & 842 & 56 & 102 \\
\hline OS2 & 6.3 & 131 & 0.56 & 0.10 & 12.20 & 1.68 & 2.82 & 0.85 & 0.64 & 0.38 & 4.69 & 6.37 & 73.63 & 844 & 62 & 94 \\
\hline OS3 & 6.9 & 58 & 1.19 & 0.16 & 8.45 & 0.25 & 1.19 & 0.88 & 0.22 & 0.23 & 2.52 & 2.77 & 90.97 & 754 & 160 & 86 \\
\hline TS1 & 6.3 & 210 & 2.16 & 0.24 & 15.94 & 0.19 & 3.96 & 0.68 & 0.56 & 0.19 & 5.39 & 5.58 & 96.59 & 750 & 158 & 92 \\
\hline TS2 & 5.5 & 216 & 2.08 & 0.14 & 13.06 & 0.12 & 4.09 & 0.82 & 0.50 & 0.16 & 5.57 & 5.69 & 97.89 & 818 & 132 & 50 \\
\hline TS3 & 5.4 & 208 & 2.22 & 0.19 & 11.20 & 0.22 & 7.04 & 0.62 & 0.44 & 0.24 & 8.34 & 8.56 & 97.43 & 832 & 118 & 50 \\
\hline
\end{tabular}

Note: Soil EC - Electrical Conductivity; SOC - Soil Organic Carbon; \% N-Total Nitrogen; A. P-Available Phosphorus; E.A - Exchangeable Acidity; ECEC-Effective Cation Exchange Capacity; Ca Calcium; Mg-Magnesium; K-Potassium; Na-Sodium; TEB - Total Exchangeable Bases; BS - Basal Saturation

Table 8: Physicochemical Properties of Soil Samples in Old Oyo National Park [Wet Season, 2017]

\begin{tabular}{|c|c|c|c|c|c|c|c|c|c|c|c|c|c|c|c|c|}
\hline \multirow[t]{2}{*}{$\begin{array}{l}\text { Soil } \\
\text { Sample }\end{array}$} & \multirow{2}{*}{$\begin{array}{l}\text { pH } \\
\left(\mathbf{H}_{2} \mathbf{O}\right. \\
1: 1)\end{array}$} & \multirow[t]{2}{*}{$\begin{array}{l}\text { Soil EC } \\
(\mu \mathrm{S} / \mathrm{cm})\end{array}$} & \multirow[t]{2}{*}{$\begin{array}{l}\text { SOC } \\
(\%)\end{array}$} & \multirow[t]{2}{*}{$\begin{array}{l}\% \\
\mathrm{~N}\end{array}$} & \multirow[t]{2}{*}{$\begin{array}{l}\text { A. P } \\
(\mathbf{m g} / \mathbf{k g})\end{array}$} & \multirow[t]{2}{*}{$\begin{array}{l}\text { E.A } \\
(\mathrm{cmol} / \mathrm{kg})\end{array}$} & \multicolumn{4}{|c|}{ Exchangeable bases $(\mathrm{cmol} / \mathrm{kg})$} & \multirow[t]{2}{*}{$\begin{array}{l}\text { TEB } \\
\text { (cmol/kg) }\end{array}$} & \multirow[t]{2}{*}{$\begin{array}{l}\text { ECEC } \\
(\mathrm{cmol} / \mathrm{kg})\end{array}$} & \multirow[t]{2}{*}{$\begin{array}{l}\text { BS } \\
(\%)\end{array}$} & \multicolumn{3}{|c|}{$\begin{array}{l}\text { Particle Size } \\
(\mathbf{g} / \mathbf{k g})\end{array}$} \\
\hline & & & & & & & Ca & $\mathrm{Mg}$ & $\mathbf{K}$ & $\mathrm{Na}$ & & & & Sand & Silt & Clay \\
\hline MS1 & 5.3 & 250 & 2.50 & 0.23 & 17.1 & 0.20 & 9.53 & 1.19 & 0.81 & 0.38 & 11.91 & 12.11 & 98.35 & 780 & 150 & 70 \\
\hline MS2 & 5.3 & 250 & 2.60 & 0.24 & 16.9 & 0.20 & 9.50 & 1.21 & 0.84 & 0.34 & 11.89 & 12.09 & 98.35 & 752 & 152 & 96 \\
\hline MS3 & 6.3 & 260 & 2.50 & 0.23 & 16.2 & 0.20 & 9.50 & 1.21 & 0.80 & 0.30 & 11.81 & 12.01 & 98.33 & 770 & 164 & 66 \\
\hline OS1 & 6.6 & 62 & 1.62 & 0.17 & 9.2 & 0.30 & 1.60 & 0.80 & 0.24 & 0.36 & 3.00 & 3.30 & 90.91 & 872 & 34 & 94 \\
\hline OS2 & 7.2 & 308 & 1.75 & 0.19 & 16.5 & 0.40 & 7.91 & 1.70 & 0.37 & 0.58 & 10.56 & 10.96 & 96.35 & 892 & 54 & 54 \\
\hline OS3 & 7.1 & 67 & 1.58 & 0.13 & 7.4 & 0.30 & 1.33 & 0.95 & 0.38 & 0.38 & 3.04 & 3.34 & 91.02 & 752 & 114 & 134 \\
\hline TS1 & 5.2 & 224 & 3.12 & 0.29 & 18.3 & 0.20 & 5.97 & 0.78 & 0.60 & 0.28 & 7.63 & 7.83 & 97.45 & 830 & 120 & 50 \\
\hline TS2 & 5.3 & 250 & 3.04 & 0.29 & 16.0 & 0.20 & 5.91 & 0.80 & 0.58 & 0.29 & 7.58 & 7.78 & 97.43 & 842 & 114 & 44 \\
\hline TS3 & 5.3 & 260 & 3.03 & 0.29 & 15.2 & 0.20 & 8.90 & 0.80 & 0.62 & 0.29 & 10.61 & 10.81 & 98.15 & 876 & 97 & 27 \\
\hline
\end{tabular}

Note: Soil EC-Electrical Conductivity; SOC-Soil Organic Carbon; \% N-Total Nitrogen; A. P-Available Phosphorus; E.A-Exchangeable Acidity; ECEC-Effective Cation Exchange; capacity; Ca Calcium; Mg-Magnesium; K-Potassium; Na-Sodium; TEB - Total Exchangeable Bases; BS - Basal Saturation 
Table 9: Physicochemical Properties of Soil Samples in Old Oyo National Park [Dry Season, 2018]

\begin{tabular}{|c|c|c|c|c|c|c|c|c|c|c|c|c|c|c|c|c|}
\hline \multirow[t]{2}{*}{$\begin{array}{c}\text { Soil } \\
\text { Sample }\end{array}$} & \multirow{2}{*}{$\begin{array}{c}\mathrm{pH} \\
\left(\mathbf{H}_{2} \mathbf{O}\right. \\
1: 1)\end{array}$} & \multirow[t]{2}{*}{$\begin{array}{l}\text { Soil EC } \\
\mu \mathrm{S} / \mathrm{cm})\end{array}$} & \multirow[t]{2}{*}{$\begin{array}{l}\text { SOC } \\
(\%)\end{array}$} & \multirow[t]{2}{*}{$\% \mathrm{~N}$} & \multirow[t]{2}{*}{$\begin{array}{c}\mathbf{A . P} \\
(\mathbf{m g} / \mathbf{k g})\end{array}$} & \multirow[t]{2}{*}{$\begin{array}{c}\text { E.A } \\
(\mathrm{cmol} / \mathrm{kg})\end{array}$} & \multicolumn{4}{|c|}{ Exchangeable bases $(\mathrm{cmol} / \mathrm{kg})$} & \multirow[t]{2}{*}{$\begin{array}{c}\text { TEB } \\
\text { (cmol/kg) }\end{array}$} & \multirow{2}{*}{$\begin{array}{c}\text { ECEC } \\
(\mathrm{cmol} / \mathrm{kg})\end{array}$} & \multirow[t]{2}{*}{ BS (\%) } & \multicolumn{3}{|c|}{$\begin{array}{c}\text { Particle Size } \\
(\mathrm{g} / \mathrm{kg})\end{array}$} \\
\hline & & & & & & & $\mathbf{C a}$ & Mg & $\mathbf{K}$ & $\mathbf{N a}$ & & & & Sand & Silt & Clay \\
\hline MS1 & 6.4 & 104 & 2.33 & 0.22 & 12.70 & 1.30 & 3.30 & 0.75 & 0.50 & 0.32 & 4.87 & 6.17 & 78.93 & 758 & 154 & 88 \\
\hline MS2 & 6.0 & 116 & 0.95 & 0.11 & 13.50 & 1.60 & 3.59 & 0.91 & 0.54 & 0.37 & 5.41 & 7.01 & 77.18 & 818 & 114 & 68 \\
\hline MS3 & 6.1 & 184 & 2.15 & 0.18 & 14.40 & 0.17 & 4.20 & 0.95 & 0.62 & 0.28 & 6.05 & 6.22 & 97.27 & 850 & 54 & 96 \\
\hline OS1 & 6.1 & 119 & 0.41 & 0.05 & 12.80 & 1.30 & 2.71 & 0.74 & 0.65 & 0.41 & 4.51 & 5.81 & 77.62 & 838 & 54 & 108 \\
\hline OS2 & 6.2 & 133 & 0.55 & 0.05 & 12.10 & 1.60 & 2.91 & 0.90 & 0.62 & 0.33 & 4.76 & 6.36 & 74.84 & 838 & 64 & 98 \\
\hline OS3 & 6.7 & 52 & 1.12 & 0.11 & 8.2 & 0.21 & 1.10 & 0.84 & 0.26 & 0.26 & 2.46 & 2.67 & 92.13 & 758 & 164 & 78 \\
\hline TS1 & 6.4 & 202 & 2.20 & 0.19 & 15.7 & 0.18 & 3.82 & 0.59 & 0.53 & 0.18 & 5.12 & 5.3 & 96.60 & 758 & 154 & 88 \\
\hline TS2 & 5.6 & 208 & 1.98 & 0.16 & 12.50 & 0.14 & 4.05 & 0.72 & 0.51 & 0.20 & 5.48 & 5.62 & 97.51 & 828 & 120 & 52 \\
\hline TS3 & 5.1 & 211 & 2.14 & 0.17 & 11.4 & 0.19 & 6.46 & 0.68 & 0.47 & 0.19 & 7.80 & 7.99 & 97.62 & 828 & 114 & 58 \\
\hline
\end{tabular}

Note: Soil EC - Electrical Conductivity; SOC - Soil Organic Carbon; \% N-Total Nitrogen; A. P-Available Phosphorus; E.A - Exchangeable Acidity; ECEC-Effective Cation Exchange Capacity; Ca -

Calcium; Mg-Magnesium; K-Potassium; Na-Sodium; TEB - Total Exchangeable Bases; BS - Basal Saturation

\begin{tabular}{|c|c|c|c|c|c|c|c|c|c|c|c|c|c|c|c|c|}
\hline \multirow[t]{2}{*}{$\begin{array}{c}\text { Soil } \\
\text { Sample }\end{array}$} & \multirow{2}{*}{$\begin{array}{c}\mathrm{pH} \\
\left(\mathbf{H}_{2} \mathbf{O}\right. \\
1: 1)\end{array}$} & \multirow[t]{2}{*}{$\begin{array}{l}\text { Soil EC } \\
(\mu \mathrm{S} / \mathrm{cm})\end{array}$} & \multirow[t]{2}{*}{$\begin{array}{l}\text { SOC } \\
(\%)\end{array}$} & \multirow[t]{2}{*}{$\% \mathbf{N}$} & \multirow[t]{2}{*}{$\underset{(\mathbf{m g} / \mathbf{k g})}{\mathbf{A . P}}$} & \multirow[t]{2}{*}{$\begin{array}{c}\text { E.A } \\
(\mathrm{cmol} / \mathrm{kg})\end{array}$} & \multicolumn{4}{|c|}{ Exchangeable bases (cmol/kg) } & \multirow[t]{2}{*}{$\begin{array}{c}\text { TEB } \\
(\mathrm{cmol} / \mathrm{kg})\end{array}$} & \multirow[t]{2}{*}{ ECEC } & \multirow[t]{2}{*}{ BS (\%) } & \multicolumn{3}{|c|}{$\begin{array}{c}\text { Particle Size } \\
(\mathrm{g} / \mathrm{kg})\end{array}$} \\
\hline & & & & & & & $\mathrm{Ca}$ & Mg & K & $\mathrm{Na}$ & & & & Sand & Silt & Clay \\
\hline MS1 & 5.8 & 260 & 2.12 & 0.28 & 13.40 & 0.60 & 5.24 & 0.94 & 0.61 & 0.44 & 7.23 & 7.83 & $\begin{array}{l}92.34 \\
\end{array}$ & 748 & 124 & 128 \\
\hline MS2 & 5.6 & 258 & 1.90 & 0.21 & 14.05 & 0.90 & 4.68 & 1.22 & 0.65 & 0.41 & 6.96 & 7.86 & 88.55 & 768 & 163 & 69 \\
\hline MS3 & 6.0 & 270 & 2.62 & 0.18 & 16.50 & 0.32 & 4.51 & 1.28 & 0.68 & 0.35 & 6.82 & 7.14 & 95.52 & 782 & 142 & 76 \\
\hline OS1 & 6.2 & 164 & 1.40 & 0.12 & 11.40 & 1.20 & 2.79 & 0.82 & 0.72 & 0.43 & 4.76 & 5.96 & 79.87 & 794 & 56 & 150 \\
\hline OS2 & 6.1 & 210 & 1.52 & 0.13 & 12.70 & 0.96 & .3 .08 & 0.94 & 0.81 & 0.38 & 5.21 & 6.17 & 84.44 & 824 & 38 & 138 \\
\hline OS3 & 6.8 & 85 & 1.20 & 0.10 & 9.10 & 0.15 & 1.86 & 1.02 & 0.32 & 0.27 & 3.47 & 3.62 & 95.86 & 734 & 96 & 170 \\
\hline TS1 & 6.2 & 228 & 2.98 & 0.27 & 15.20 & 0.12 & 3.74 & 0.63 & 0.52 & 0.20 & 5.09 & 5.21 & 97.70 & 814 & 118 & 68 \\
\hline TS2 & 5.7 & 224 & 1.78 & 0.23 & 13.60 & 0.12 & 4.98 & 0.76 & 0.56 & 0.24 & 6.54 & 6.66 & 98.20 & 864 & 120 & 16 \\
\hline TS3 & 5.3 & 232 & 2.50 & 0.31 & 15.20 & 0.11 & 7.30 & 0.72 & 0.51 & 0.21 & 8.74 & 8.85 & 98.76 & 858 & 72 & 70 \\
\hline
\end{tabular}

Note: Soil EC - Electrical Conductivity; SOC - Soil Organic Carbon; \% N - Total Nitrogen; A. P - Available Phosphorus; E.A - Exchangeable Acidity; ECEC - Effective Cation Exchange Capacity; Ca -

Calcium; Mg - Magnesium; K - Potassium; Na - Sodium; TEB - Total Exchangeable Bases; BS - Basal Saturation

Table 11: Mean values of physicochemical parameters of soil samples of Old Oyo National Park

\begin{tabular}{|c|c|c|c|c|c|c|}
\hline \multirow[b]{2}{*}{ Parameters } & \multicolumn{4}{|c|}{ Mean Values \pm Standard Deviation } & \multirow[t]{2}{*}{ Critical Limits } & \multirow[t]{2}{*}{ Reference } \\
\hline & $\begin{array}{l}\text { Dry Season } \\
\text { (2017) }\end{array}$ & $\begin{array}{l}\text { Wet Season } \\
\text { (2017) }\end{array}$ & $\begin{array}{l}\text { Dry Season } \\
\text { (2018) }\end{array}$ & $\begin{array}{l}\text { Wet Season } \\
\text { (2018) }\end{array}$ & & \\
\hline $\mathrm{pH}$ & $6.12 \pm 0.48$ & $5.96 \pm 0.84$ & $6.07 \pm 0.47$ & $5.97 \pm 0.43$ & $3.0-8.5$ & NMSU, 2000 \\
\hline Soil EC $(\mu \mathrm{S} / \mathrm{cm})$ & $150.11 \pm 56.98^{\mathrm{ab}}$ & $214.56 \pm 87.87^{\mathrm{ac}}$ & $147.67 \pm 55.93^{\text {cd }}$ & $214.56 \pm 58.05^{\mathrm{bd}}$ & - & NMSU, 2000 \\
\hline $\operatorname{SOC}(\%)$ & $1.59 \pm 0.79^{\mathrm{a}}$ & $2.42 \pm 0.62^{\mathrm{ab}}$ & $1.54 \pm 0.77^{\mathrm{b}}$ & $2.00 \pm 0.60$ & - & - \\
\hline$\% \mathrm{~N}$ & $0.16 \pm 0.06^{\mathrm{a}}$ & $0.23 \pm 0.06^{\mathrm{ab}}$ & $0.14 \pm 0.06^{\mathrm{bc}}$ & $0.20 \pm 0.08^{c}$ & $0.05-0.15$ & Tisdale et al., 1993 \\
\hline A.P $(\mathrm{mg} / \mathrm{kg})$ & $12.68 \pm 2.08$ & $14.76 \pm 3.78$ & $12.59 \pm 2.08$ & $13.46 \pm 2.22$ & $8-20$ & Rankine and Fairhurst, 1999 \\
\hline E.A $(\mathrm{cmol} / \mathrm{kg})$ & $0.87 \pm 0.0 .82^{\mathrm{a}}$ & $0.24 \pm 0.07^{\mathrm{a}}$ & $0.74 \pm 0.68$ & $0.50 \pm 0.43$ & - & - \\
\hline $\mathrm{Ca}(\mathrm{cmol} / \mathrm{kg})$ & $3.74 \pm 0.1 .58^{\mathrm{a}}$ & $6.68 \pm 3.28^{\mathrm{ab}}$ & $3.57 \pm 1.43^{\mathrm{b}}$ & $4.24 \pm 1.60$ & - & - \\
\hline $\mathrm{Mg}(\mathrm{cmol} / \mathrm{kg})$ & $0.83 \pm 0.13^{\mathrm{a}}$ & $1.05 \pm 0.31^{\mathrm{ab}}$ & $0.79 \pm 0.12^{b}$ & $0.93 \pm 0.22$ & $0.08-0.25$ & Rankine and Fairhurst, 1999 \\
\hline $\mathrm{K}(\mathrm{cmol} / \mathrm{kg})$ & $0.57 \pm 0.18$ & $0.58 \pm 0.22$ & $0.52 \pm 0.12$ & $0.60 \pm 0.14$ & $0.20-0.40$ & Rankine and Fairhurst, 1999 \\
\hline $\mathrm{Na}(\mathrm{cmol} / \mathrm{kg})$ & $0.31 \pm 0.11$ & $0.36 \pm 0.09$ & $0.28 \pm 0.08$ & $0.33 \pm 0.10$ & $10-30$ & NMSU, 2000 \\
\hline TEB $(\mathrm{cmol} / \mathrm{kg})$ & $5.45 \pm 1.55^{\mathrm{a}}$ & $8.67 \pm 3.61^{\mathrm{abc}}$ & $5.16 \pm 1.41^{\mathrm{b}}$ & $6.09 \pm 1.59^{c}$ & - & \\
\hline ECEC & $6.32 \pm 0.1 .62^{\mathrm{a}}$ & $8.91 \pm 3.58^{\mathrm{abc}}$ & $5.91 \pm 1.45^{\mathrm{b}}$ & $6.59 \pm 1.58^{c}$ & $2-12$ & NMSU, 2000 \\
\hline BS (\%) & $86.67 \pm 11.52^{\mathrm{a}}$ & $96.26 \pm 3.07^{\mathrm{ab}}$ & $87.74 \pm 10.24^{b}$ & $92.36 \pm 6.70$ & - & - \\
\hline Sand $(\mathrm{g} / \mathrm{kg})$ & $808.00 \pm 40.66$ & $818.44 \pm 55.83$ & $808.22 \pm 38.67$ & $798.44 \pm 45.63$ & - & - \\
\hline Silt $(\mathrm{g} / \mathrm{kg})$ & $112.00 \pm 44.11$ & $111.00 \pm 43.99$ & $110.22 \pm 43.74$ & $103.22 \pm 41.11$ & - & - \\
\hline Clay $(\mathrm{g} / \mathrm{kg})$ & $80.00 \pm 19.95$ & $70.56 \pm 32.68$ & $81.56 \pm 18.99$ & $98.33 \pm 50.15$ & - & - \\
\hline
\end{tabular}

Note: Means with the same alphabets are significantly different at $P \leq 0.05$ 


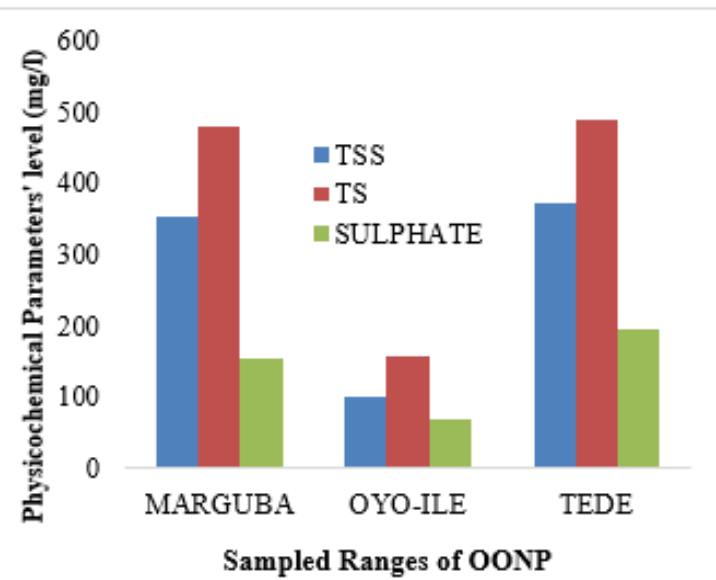

Fig 1: Mean plot of water physicochemical parameters (above permissible limit) among the selected ranges of Old Oyo National Park

The mean values of EC of the water samples were higher during the dry season. This is contrary to Ajibade et al. (2008) who reported higher values during the wet season due to the leaching of the mineral salt from the bedrock and re-suspension of solids. The high EC recorded in the water samples is an indication of high dissolved salts and could be linked to discharge of sewage materials and leaching of inorganic contaminants. The mean values of TDS of the water samples were higher during the dry season. This could be as a result of the tidal influence of the rivers during the wet season. The low TDS values observed during the wet season of 2017 may be due to dilution and usage by phytoplankton (Adakole et al., 2008). The quantity of TDS is often proportional to the degree of pollution and further indicates the salinity behaviour of river water (Masood et al., 2015). The alkalinity values gotten from this study are higher than those reported by Omonona et al. (2018) at Omo Forest Reserve. The mean values of alkalinity obtained in this study are higher during the wet season and may be due to high decomposition of organic matter. The mean values of TSS of the water samples were higher during the dry season. The very high values recorded from Rivers Oopo and Sooro in the dry season of 2018 may be attributed to atmospheric particle deposits and storm water run-off. The variations in $\mathrm{pH}$ may have caused some solutes to precipitate or probably affect the solubility of the suspended mater (Bellingham, 2012). The mean values of nitrate in the water samples were higher during the wet seasons. This may be due to contributory run-off of chemical fertilizers and from oxidation of nitrogenous waste products in human and animal faeces into the rivers sampled as corroborated by Dami et al. (2013). The low nitrate concentration recorded in this study is typical of surface waters (CCME, 2009) but can reach high levels from agricultural runoff, or from contamination by human or animal wastes. The mean values of phosphate in the water samples were higher during the wet seasons. This may be due to domestic and industrial discharges or agricultural run-off from agricultural farms in the surrounding communities and other phosphate sources. The low phosphate concentration recorded in this study may be attributed to dilution and movement of water which could not allow aquatic sedimentation and decay of organic matter (Keke et al., 2015). The mean values of sulphate in the water samples were higher during the dry seasons. The values obtained during the dry season of 2017 showed that the sulphate content of all the rivers sampled were above the NSDWQ (2007) permissible limit while those of Rivers Ogun, Ayinta, Tessi and Sooro were above the WHO (2011) permissible limit. The presence of sulphate in the sampled rivers may be attributed to the washing activities from surrounding communities and discharge of house hold effluents into the rivers. Furthermore, the mean values of chloride in the water samples were slightly higher during the dry seasons. This may be due to the concentration of this anion from excessive water evaporation from the rivers as corroborated by Oyhakilome et al. (2012). High concentration of chloride is considered to be the indicator of pollution due to organic wastes of animal origin, regarded harmful to aquatic life and troublesome in irrigation water (Rajkumar et al., 2004). The mean values of DO in the water samples were higher during the wet seasons. The lower values obtained during the dry seasons may be due to lower water depth and less agitation by wind current (Ajibade et al., 2008). The DO is very crucial for the survival of aquatic life and it is also used to evaluate the degree of freshness of a river (Andem et al., 2012). The mean values of the BOD in the water samples were slightly higher during the wet seasons. This may probably be due to the increased input of decomposable organic matter into the rivers sampled through surface run-off. High BOD is an indication of poor water quality, and the lower the BOD, the less organic matter present in water (Samuel et al., 2015). The mean values of the COD in the water samples were higher during the wet seasons. The high values of COD obtained from the study may be as a result of chemical oxidation of some organic substances which are oxidized biologically (Okoroafor et al., 2013) due to discharges of domestic wastewater from nearby settlements, surface and ground water carrying chemicals directly from agricultural farms (Abolude $e t$ al., 2013).

Soil $\mathrm{pH}$ observed in this study were slightly acidic based on the ratings of Agbede (2008) though below the $\mathrm{pH}$ range of 6.8 to 8.0 recommended for optimum 
plant's growth (Jain et al., 2015). Soils with low pH have been reported to favour availability, mobility and redistribution of metals due to increased solubility of the ions in acidic environment (Oviasogie and Ndiokwere, 2008). The higher values observed in the wet seasons may be due to the fact that the basic cations were forced off the soil colloids by the mass action of hydrogen ions from the rain as those attached to the colloids (Edori and Iyama, 2017). High conductivity values observed in the soil samples is an indication of anthropogenic interference and infer the availability of soluble salts or ions in the soil samples as corroborated by Arias et al. (2005) and Egbenda et al. (2015). Jain et al. (2015) reported that low soil EC is often appropriate for plant growth. Suitable amount of soil $\mathrm{pH}$ and EC leads to optimum availability of nutrients, reduced accessibility of toxic elements and increased activity of micro-organisms (Raman and Sathiyanarayanan, 2009). The SOM observed in the study is greater than $2.0 \%$ asserted by Ayolagha and Onwugbuta (2001) to be conducive for heavy metal chelation formation in soils. Much of the soil organic matter (SOM) was composed of soil organic carbon (SOC). This may be due to the large percentage of carbon in plant tissues as corroborated by Havlin et al. (2005). Mandal et al. (2014) also reported that forest soil reserves much higher organic carbon including varying proportion of active organic carbon fractions and stable organic matter, referred to as humus in comparison to agriculture and other land use. The soils of the park are considered very good since nitrogen value greater than $0.1 \%$ is rated good (Defoer et al., 2000). The soils studied are not deficient in available phosphorus because the values were generally higher than $6.0 \mathrm{mg} / \mathrm{kg}$ (Defoer et al., 2000). This may be due to the availability of high amount of organic matter and plants decomposition (Ideriah et al., 2006) and leaching off of fertilizer nutrients from agricultural farms from the surrounding communities. The high levels of $\mathrm{Ca}$ and $\mathrm{Mg}$ in the soils could be attributed to the high base saturation and $\mathrm{pH}$ levels of the soils studied (Middha et al., 2015). The variation in the $\mathrm{K}$ content of the soils across the seasons of sampling may be due to soil saturation which resulted in widening of clay minerals, releasing previously fixed $\mathrm{K}$ as posited by Middha et al. (2015). The $\mathrm{Ca}, \mathrm{Mg}$ and $\mathrm{K}$ concentration of the soils was similar to that reported by Uzoho et al. (2007) for most Nigerian soils. This was attributed to the leaching of nutrients induced by high rainfall conditions. There was variation in the effective cation exchange capacity of the soils. However, they were within the critical range devised for ecological zones (NMSU, 2000). The concentration of $\mathrm{Na}$ was observed to be the lowest of the exchangeable bases. High $\mathrm{Na}$ concentration has been reported to pose a threat on soil permeability, soil texture and also reduces the soil's water intake (Patil et al., 2014). The ECEC gives the soil a buffering capacity which may slow down the leaching of nutrient cations and positively charged contaminants because they affect both soluble and exchangeable metal levels (Yoo and James, 2002). The mean range of BS observed in this study was higher than the 72.32 - 97.35 reported by Alarape (2002). The base saturation of the soils studied was also higher than the maximum critical level established for ecological zone as reported by Holland et al. (1989). The particle size distribution of the sampled soils showed that the soils of Old Oyo National Park had high sand content. Even though this may allow high permeability of water and leachates, it may also aid environmental contamination. Nyles and Ray (1999) had earlier reported that soils possessing separate high sand and low clay content have high pollutant leaching potentials. The physicochemical properties of soil, such as texture, cation exchange capacity, $\mathrm{pH}$ and the amount of organic matter within the soil, are important parameters that affect the heavy metal accumulation rate of soils (Wua and Zhang, 2011).

Conclusion: The marked variation and significant differences in some physicochemical parameters observed in this study indicate different environmental conditions. The sampled waterholes (rivers) are of poor quality while their polluted nature may have implications on human and wild animal health in the park as it serves as a source of drinking water. The soil physicochemical parameters above comparable critical limits and observed seasonal variability may have elicited from the impact of anthropogenic activities by the surrounding communities. This in turn can most likely have an impact on the park's ecosystem.

\section{REFERENCES}

Abolude, DS; Barak, Z; Tanimu, Y; Bingari, MS; Opabunmi, OO; Okafor, DC (2013). Assessment of the Concentration of Metals in Sewage Treatment Pond of the Ahmadu Bello University Zaria, Nigeria. J. Aquat. Sci. 28(1):24-34

Adakole, JA; Abulode, DS; Balarabe, ML (2008). Assessment of Water Quality of a Man-Made Lake in Zaria Nigeria. Sengupta, M. and Dalwani, R., Eds., Proceedings of Taal 2007: The 12th World Lake Conference, 1373-1382

Adetoro, AO; Oyeleye, DO; Ijeomah, HM (2011). Causes and impacts of conflicts on biodiversity management at the Buffer zone of Old Oyo National Park, Oyo State, Afri. Res. Rev. 5 (1):485 $-491$ 
Agbede, OO (2008). Soil husbandry: life for national food security and economic empowerment. An Inaugural Delivered on The 19th of March, 2008 at Nasarawa State University Keffi, Nigeria.

Ajibade, WA; Ayodele, IA; Agbede, SA (2008). Water quality parameters in the major rivers of Kainji Lake National Park, Nigeria. Afr. J. Environ. Sci. Technol. Vol. 2 (7), pp. 185-196

Akinyemi, AF; Kayode, IB (2010). Effects of Agricultural Practices on the Distribution of Western Hartebeest (Alcelaphus buselaphus) in Old Oyo National Park, Nigeria. J. Environ. Issues Agric. in Dev. Count. Vol. 2 (1)121-130

Alarape, A (2002). Culture and Conservation in and around Old Oyo National Park. Ph.D. Thesis Submitted to the Department of Wildlife and Fisheries, University of Ibadan.

Alsumaiti, TS; Shahid, SA (2018). Comprehensive Analysis of Mangrove Soil in Eastern Lagoon National Park of Abu Dhabi Emirate. Int. J. Bus. Appl. Soc. Sci., 4(5): 39-56.

Andem, AB; Udofia, U; Okorafor, KA; Okete, JA; Ugwumba, AAA (2012). A Study on Some Physical and Chemical Characteristics of Ona River, Apata, Ibadan South-west, Oyo State, Nigeria, Eur. J. Zool. Res., 1 (2):37-46.

Arias, ME; Gonzalez-Perez, JA; Gonzalez-Villa, FJ; Ball, AS (2005). Soil health: A new challenge for microbiologist and chemists. Int. Microbiol. 8: 13-21.

Ayoade, AA; Fagade, SO; Adebisi, AA (2006). Dynamics of Limnological Features of Two ManMade Lakes in Relation to Fish Production. Afr. J. Biotechnol. 5(10): 1013-1021.

Ayolagha, GA; Onwugbata, GC (2001). Suitability comparison of waste disposal site. 27th Proceedings of the Annual Conference of the Soil Science of Nigeria. November 5-9, 2001, University of Calabar, Nigeria.

Bellingham, K (2012). Physicochemical Parameters of Natural Waters. Stevens Water Monitoring Systems, Inc. (http://www.stevenswater.com)

Bremmer, JM (1996): Nitrogen - Total. In: Methods of Soil Analysis, Part 3, Chemical Method. 2nd Edition, ed., Sparks, D. L., Soil Science Society of America Book Series, No. 5. SSSA, Madison, WI. USA, pp.1085-1121.

Canadian Council of Ministers of the Environment [CCME], (2009) Nitrate and Nitrite, http://www.ccme.ca/sourcetotap/nitrates.html

Chaudhari, KG (2013). Studies of the physicochemical parameters of soil samples. $A d v$. Appl. Sci. Res. 4(6): 246 - 248

Chen, JC; Lin, CY (1995). Responses of Oxygen Consumption, Ammonia-N Excretion and Urea-N Excretion of Panaeus chinensis Exposed Ambient Ammonia at Different Salinity and pH Levels. $J$. Aquaculture. 136: 243-255

Dami, A; Ayuba, HK; Amukali, O (2013). Groundwater pollution in Okpai and Beneku, Ndokwa East local Government, Delta State, Nigeria. J. Env. Res. Mgt. 4(1):0171- 0199.

Dar, ZA; Qadri, H; Mir, SA; Beigh, B; Amin, A (2018). Assessment of Physico-Chemical Parameters of Forest Soil of Hirpora Wildlife Sanctuary, Kashmir. Int. J. Adv. Res. Sci. Eng. 7(4): 46-56

Davies-Colley, RJ (2013). River water quality in New Zealand: an introduction and overview. In Dymond, J. R. ed. Ecosystem services in New Zealand - conditions and trends. Manaaki Whenua Press, Lincoln, New Zealand.

Deepak, S; Singh, NU (2014). The Relationship between Physico-chemical Characteristics and Fish Production of Mod sagar Reservoir of Jhabua District, MP, India. Res. J. Recent Sci. 3, 82-86

Defoer, T; Budelman, A; Toulmin, C; Carter, SE (2000). Building common knowledge: participatory learning an action research (part I). In: T. Defoer \& A. Budelman, (Eds). Managing soil fertility in the tropics. A resource guide for participatory learning and action research. Amsterdam: The Netherlands: Royal Tropical Institute.

Diersing, N (2009). Water quality frequently asked questions. Florida Books National Marine Sanctuary. Key West, Florida.

Edori, OS; Iyama, WA (2017). Assessment of Physicochemical Parameters of Soils from Selected Abattoirs in Port Harcourt, Rivers State, Nigeria. J Environ Anal Chem. 4: 194 
Egbenda, PO; Thullah, F; Kamara, I (2015). A physicochemical analysis of soil and selected fruits in one rehabilitated mined out site in the Sierra Rutile environs for the presence of heavy metals: Lead, Copper, Zinc, Chromium and Arsenic. Afri. J. of Pure and Appl. Chem. 9(2):2732.

Furtak, K; Gajda, AM (2018). Biochemical methods for the evaluation of the functional and structural diversity of microorganisms in the soil environment. Post. epy Mikrobiologii 57: 194 202.

Furtak, K; Grzadziel J; Anna Gałazka, A; Jacek Niedzwiecki, J (2019). Analysis of Soil Properties, Bacterial Community Composition, and Metabolic Diversity in Fluvisols of a Floodplain Area. Sustainability 11, 3929;

Gee, GW; Or, D (2002): Particle size analysis. In: Dane, J., and Topp, G. C., eds., Methods of Soil Analysis, Part 4: Physical Methods. Soil Science Society of America, Madison, WI, pp. 255-294.

Gothwal, R; Gupta, GK (2019). Physico-Chemical Analysis of Soil during Summer Season in Lentic Fresh Water Ecosystem: Nakki Lake-Mount Abu (Rajasthan), India. World Sci. News 115: 117-127

Havlin, JL; Beaton, JD; Tisdale, SL; Nelson, WL (2005). Soil Fertility and Nutrient. Management: An Introduction to Nutrient Management. $7^{\text {th }}$ Edition.Pearson/Prentice Hall. Upper Saddle River, NJ.

Holland, MD; Allen, VG; Burton, D; Urphy, ST (1989). Land Evaluation and Agricultural Recommendations, Cross River National Park, Oban Division prepared by Oversea Development Natural Resources Institute in collaboration with WWF for the Federal Republic of Nigeria and the Cross Rivers State Government.

Ideriah, TKJ; Omuaru, VOT; Adiukwu, PA (2006). Soil quality around a waste dumpsite in Port Harcourt, Nigeria. Afr. J. Ecol. 44: 388-394

Jackson, ML (1958). Soil Chemical Analysis, In: N. J. Englewood (Ed.) (2nd ed.), Printice-Hall Inc. pp 59-67.

Jain, S; Middha, R; Juneja, SK; Kaushik, P (2015). Comparative Physicochemical Analysis of Restored and Unrestored Soils of Village Thooni Ram Laxmanpura of Chaksu Block, Jaipur, Rajasthan. Int. J. Curr. Res. 7(5):15706-15710.
Keke, UN; Arimoro, FO; Ayanwale, AV; Aliyu, SM (2015). Physicochemical Parameters and Heavy Metals Content of Surface Water in Downstream Kaduna River, Zungeru, Niger State, Nigeria. Appl. Sci. Res. J., Vol. 3(2): 46 -57

Lemaire, G; Franzluebbers, A; Carvalho, PC; Dedieu, B (2014). Integrated crop-livestock system: Strategies to achieve synergy between agricultural production and environmental quality. Agric. Ecosyst. Environ. 190: 4-8

Mandal, UK; Venkanna, K; Raju, AJS; Sharma, KL; Adake, RV; Pushpanjali, RBS; Babu, BP (2014). Carbon stocks in major soil types and land-use systems in semiarid tropical region of southern India. Curr. Sci. 106 (4): 604-611

Martinez, FB; Mijares, MBB; Galera, IC (2011). Assessment of the water quality of Mamba River of Mts. Palaypalay/Mataas na Gulod, Southern Luzon, Phillipines. 2011 International Conference on Chemistry and Chemical Process IPCBEE vol. 10, IACSIT Press, Singapore

McLean, EV (1982). Aluminium, In: Methods of soil analysis, Part 2, eds. Page, A. L., Miller, R. H. and Keeney, D. R. American Society of Agronomy, Madison WI., pp. 978-998.

Middha, R; Jain, S; Juneja, SK (2015). A comparative study of physic-chemical parameters of restored and unrestored soils of two villages of Chaksu Block, Jaipur, Rajasthan. Int. J. Eng. Technol. Sci. Res. 2: 9-13

Nelson DW; Sommers, LE (1982): Total carbon, organic carbon and organic matter. In: Methods of soil analysis, part 2, eds. Page, A. L., Miller, R. H. and Keeney, D. R. American Society of Agronomy, Madison WI., pp. 539-579.

New Mexico State University (NMSU). (2000). Critical values of soil fertility indices for tropical soils. New Mexico State University Press. Pp 42

NSDWQ (2007). Nigeria Standard for Drinking Water Quality, Nigeria Industrial Standard, Approve by Standard Organization of Nigeria Governing Council. 20: 15-19.

Nyles, CB; Ray, RN (1999). The Nature and Properties of Soils. 12th Ed. United States of America. pp. 743 - 785 
Okoroafor, KA; Effanga, EO; Andem, AB; George, UU; Amos, DI (2013). Spatial Variation in Physical and Chemical Parameters and MacroVertebrates in the Intertidal Regions of Calabar River, Nigeria. Greener J. Geol. Earth Sci. 1(2): 063-072

Oladeji, SO; Agbelusi, EA; Ajiboye, AS (2012). Assessment of Aesthetic Values of Old Oyo National Park. Am. J. Tour. Manag. 1(3): 69-77

Olukunle, O (2000). Homestead Pond Management. Published by John Archers (Publishers) Ltd. Ibadan, Pp. 34

Omonona, AO; Ajani, F; Adetuga, AT; Koledoye, OJ (2018). Assessment of heavy metal contamination in soil and water samples in Omo Forest Reserve, Nigeria. Afr. J. Biom. Res. 22 (2): 207-214

Oviasogie, PO; Ndiokwere, CL (2008). Fractionation of lead and cadmium in refuse dump soil treated with cassava milling effluent. J. Agric. Environ. 9:10.

Oyeleke, OO; Odewumi, OS; Mustapha, RA (2015). Assessment of Management Practices for Ungulates in Old Oyo National Park, Nigeria. EJESM 8(5):548-555

Oyhakilome, GI; Aiyesanmi, AF; Akharaiyi, FC (2012). Water Quality Assessment of the Owena Multi-Purpose Dam, Ondo State, Southwestern Nigeria. J. Environ. Prot. 3. 14-25

Patil, PN; Sawant, DV; Deshmukh. RN (2012). Physicochemical parameters for testing of water A review. Int. J. Environ. Sci. 3(3): 1194-1206

Perlman, H (2013). Water Temperature. USGS Science for the Changing World. The USGS Water Science School. U.S. Department of the Interior/US. Geological Survey.
Rajkumar, S; Velmurugan, P; Shanti, K; Ayyasamy, PM; Lakshman, AP (2004). Water quality of Kodaikanal Lake, Tamilnadu in relation to physicochemical and bacteriological characteristics. Capital Publishing Company. pp. 339-246.

Raman, N; Sathiyanarayanan, D (2009). Physicochemical Characteristics of Soil and Influence of Cation Exchange Capacity of Soil in and Around Chennai. Rasayan J. Chem. 2(40): 875-885.

Samuel, PO; Adakole, JA; Suleiman, B (2015). Temporal and Spatial Physicochemical Parameters of River Galma, Zaria, Kaduna State, Nigeria. Resour. Environ. 5(4): 110-123

Swaleh, SB; Usmani, N (2016). Impact of heavy metals in environment: A review. Int. J. Innov. Res. Sci. Eng. Technol. 5 (9): 15968-15975

Udo, EJ; Ibia, TO; Ogunwale, JA; Ano, AO; Esu, IE (2009). Manual of Soil, Plant and Water Analysis. Sibon Books Ltd., Lagos. 183pp.

Uzoho, BU; Oti, NN; Ngwuta, A (2007). Fertility status under land use types on soils of similar lithology. J. Am. Sci., 3(4):20 - 29.

Wua, F; Zhang, G (2011). The influence of $\mathrm{pH}$ and organic matter content in paddy soil on heavy metal availability and their uptake by rice plants. Environ Pollut. 159:84-91

Yoo, MS; James, BR; (2002). Zinc extractability as a function of $\mathrm{pH}$ in organic waste-contaminated soils. Soil Sci. 167: 246-259 